\title{
Mass transfer enhancement produced by laser induced cavitation
}

Peter R. Birkin ${ }^{* a}$, Hanne-Maria Hirsimäki ${ }^{a b}$, Jeremy G. Frey ${ }^{a}$, Timothy G. Leighton $^{b}$ ${ }^{\mathrm{a}}$ School of Chemistry, ${ }^{\mathrm{b}}$ Institute of Sound and Vibration Research

University of Southampton

Southampton

SO17 1BJ

United Kingdom

Abstract

A microelectrode is used to measure the mass transfer perturbation and characteristics during the growth and subsequent collapse of a single bubble (which following its initial expansion, achieved a maximum radius, $R_{m}$, of $\sim 500-1000 \mu \mathrm{m}$ ). This mass transfer enhancement was associated with the forced convection, driven by bubble motion, as the result of a single cavitation event generated by a laser pulse beneath a $25 \mu \mathrm{m}$ diameter $\mathrm{Au}$ microelectrode. Evidence for bubble growth and rebound is gained from the electrochemical and acoustic measurements. This is supported with high-speed video footage of the events generated. A threshold for the formation of large cavitation bubbles in electrolyte solutions is suggested.

Keywords: cavitation, laser, mass transfer, pressure signal, high-speed imaging.

${ }^{*}$ Corresponding author. Tel: + 442380 594172, Fax: + 442380 593781, e-mail: prb2@ soton.ac.uk 


\section{Introduction}

The parasitic generation of cavitation can be extremely costly for industry. The process termed 'cavitation' has been extensively investigated since its discovery around the beginning of the $20^{\text {th }}$ century [1]. Cavitation may be defined as the rapid expansion and collapse of bubbles or voids within a liquid media. Cavitation can be generated by a number of different techniques including ultrasound, hydrodynamic flow and laser pulse within a liquid. However, it is important to note that the generation of each cavitation event can differ depending on the technique employed. For example cavitation bubbles generated in liquids by power ultrasound or hydrodynamic flow are usually formed through the suitable excitation (e.g. local pressure conditions) of pre-existing nuclei within the liquid. These nuclei can be free floating or attached to a solid/liquid interface. The cavitation bubbles that form under these conditions grow under tension and then can collapse providing that the physical conditions are suitable. In turn the maximum size attained by the bubble at the end of the growth phase is related to the local conditions within the liquid. For example the maximum dimension achieved by bubbles, which are initially $\sim 1-10 \mu \mathrm{m}$ in radius, can be as great as $\sim 100 \mu \mathrm{m}$ [2] to even $\sim 1000 \mu \mathrm{m}$ [3] (to cite example studies using ultrasonic horns [2] and hydrodynamic flow [3]). This range of sizes, and the unpredictability of the times and locations of cavitation nucleation, hinders examination of individual hydrodynamic or ultrasonic cavitation events: measurement of individual events may be required to validate models, or to correlate the results of one sensor with those of another, as is done in this paper with mass transfer sensing and high-speed photography. Furthermore hydrodynamic or ultrasonic cavitation 
tends to produce clusters [4-6] and clouds of events within a particular reactor. While this may be useful for bulk processing of materials, it makes the study of the cavitation processes that are occurring more difficult as the locality of each event is hard to predict and interactions between bubbles (cluster effects) may also occur. In contrast to this, the generation of laser induced cavitation relies on a focused pulsed beam which provides sufficient energy densities to break the liquid down into a plasma [7]. This region then expands and cools to form a large (of mm dimensions) single cavity within the liquid. The collapse of this bubble then occurs as the forces acting on the bubble drive it to implode. Our understanding of cavitation has been significantly enhanced by the study of these single bubbles created by large energy densities $\left(>10^{10} \mathrm{~W} \mathrm{~cm}^{-2}\right)$ [7] into a suitable medium [8-11]. These single cavitation events can, as well as being generated by laser pulses, be produced through spark discharge into a liquid [12]. A significant advantage of these techniques is that they are used to produce a sufficiently large (mm dimensions) cavitation event for imaging with high-speed photography at a known location. One such study of cavitation events was reported by Philipp and Lauterborn [11]. They detailed an extensive investigation of the damage to a surface produced by single cavitation bubbles $\left(R_{\max }\right.$ typically $1.45 \mathrm{~mm}$ ) produced by laser action [11]. They showed, using high frame rate photography techniques (frame rates up to $10^{6}$ frames per second) and ex-situ microscopy of various solid surfaces, that the damage caused by one or more (up to 5000) single bubble collapses depended strongly on the dimensionless distance between the bubble and the solid liquid interface (defined as $s / R_{\max }$ where $s$ and $R_{\max }$ are the distance between the bubble centre and the solid interface and the maximum bubble radius respectively). These authors showed that most surface damage 
associated with single bubble collapse originates from shock wave emission. This emission originates from the collapse phase of the bubble and is often associated with the generation of a toroidal bubble as the result of microjet formation. Surface damage, as the result of microjet impingement on the surface, was not found to be extensive. Philipp and Lauterborn also showed that, even though the scale of the damage they observed was of the order of $\mu \mathrm{m}$ in depth, the surface damage areas were very complex in shape. However, this and previous studies have relied on the ex-situ investigation of surface damage as the result of microjet or shock wave impact to ascertain the individual effect of bubbles on surfaces. Furthermore these ex-situ investigations were time integrated such that they examine only the cumulative result of the entirety of each erosion event. While a great deal of important information has been gathered in this manner, in situ investigations of surface effects and mass transfer of material in the liquid have not been detailed to such an extent. In order to gain further insight into these effects, and in particular the mass transfer of material from a single cavitation event, an electrochemical investigation has been undertaken. This approach has the additional advantage that the mass transfer (or erosion) can be time resolved and correlated to the bubble dynamics (or even, as will be shown later, the laser pulse).

Electrochemical investigations of cavitation date back to the 1930's [13]. However, most of these studies have focussed on bulk effects of cavitation produced by ultrasound. While this has merit in enabling study of the effects of cavitation clouds on electrochemical processes (including bulk electrolysis, materials deposition and electroanalytical processes [14-18] etc), it does not allow the individual effects of single cavitation bubbles to be determined. Such analysis of single events is 
important, as it can be used to validate CFD and other models of the system. However, single event interrogation within a cavitation cloud is possible using a microelectrode technique. Here the microelectrode can be placed within a cavitating environment, and the perturbation of the mass transfer characteristics of the solution determined under steady state conditions [19-21]. In such a technique the solution contains a redox active material and the electrode is maintained under mass transfer limited conditions. Each individual bubble collapse or motion will be registered as a current time transient at the microelectrode. This idea can be extended to the study of the erosive nature of individual cavitation bubbles [22-24]. In this case a passivated electrode surface is maintained under electrochemical control. Erosion due to cavitation above a passivated surface results in fresh deposition of material to 'heal' or repassivate the electrode surface. Hence individual cavitation erosion events are detected as a series of anodic current time transients. This technology can then be used to study material effects or determine the local effects of single cavitation events. This is because there are a number of mechanisms induced in a cavitation environment which can cause mass transfer enhancements as a result of forced convection [19, 25]. However, surface erosion is strongly related to transient or inertial collapse. Clearly distinguishing between these mechanisms requires local knowledge of both mass transfer and surface erosion. This has recently been achieved through the deployment of a novel dual microelectrode into a cavitation environment $[23,26-28]$.

While the electrochemical effects of cavitation have been extensively studied in relation to bubbles generated through an ultrasonic sound field, the study of laser induced cavitation has yet to be reported using this microelectrode approach. 
Considering the potential benefits of this approach, it would seem desirable to perform studies in this area. The following manuscript reports preliminary results obtained from an investigation of cavitation produced by a laser pulse. In this arrangement a large (maximum bubble radius, $R_{m}, \sim 0.5-1.0 \mathrm{~mm}$ ) single bubble is formed by a single Nd:YAG laser pulse ( $5 \mathrm{~ns}$ ) focussed inside an electrochemical cell.

The following details the results and findings of a preliminary electrochemical investigation of the mass transfer effects of laser induced cavitation. 


\section{Experimental}

Cavitation bubbles were generated by focusing an electro-optically Q-switched Nd:YAG laser (Continuum SL II-10, Photonics Solutions PLC) into an electrochemical cell. The laser produced $5 \mathrm{~ns}$ pulses at the wavelength of $532 \mathrm{~nm}$ and delivered energies up to $170 \mathrm{~mJ}$ per pulse. The beam diameter was $6 \mathrm{~mm}$. The beam was typically first expanded with a concave lens $\mathrm{L}_{3}$ (Comar optics, $f=-40 \mathrm{~mm}$, where $f$ represents the focal length), then collimated with a convex lens $\mathrm{L}_{2}$ (Comar optics, $f=150 \mathrm{~mm}$ ) and finally focused with another convex lens $\mathrm{L}_{1}$ (Mellesgriot, $f=$ $18 \mathrm{~mm}$ ) that was mounted onto the front window of the electrochemical cell (see figure 1 frame (u)). The electrochemical cell had four windows, access for the working electrode and hydrophone from the top and separate holders for the reference and counter electrode. The laser power was adjusted by changing the Q switch delay. A powermeter (Coherent Fieldmaster) was employed to measure the applied power as a function of the Q switch setting.

A $25 \mu \mathrm{m}$ diameter Au microelectrode sealed in glass was constructed and used as the working electrode [29]. The diameter of the electrodes fabricated this way was very small $(\sim 2.3 \mathrm{~mm})$. Platinum gauze served as the counter electrode and a saturated calomel electrode (SCE) was used as the reference.

The position of the working electrode was controlled by micropositioner (Time and Precision). The micropositioner had manual $25 \mathrm{~mm} \mathrm{X}$ and $\mathrm{Y}$ stages and a motorised $50 \mathrm{~mm} \mathrm{Z}$ stage, all with $10 \mu \mathrm{m}$ resolution. The stepper motor was controlled by stepper drive (Parker automation) and in-house written software. The micropositioner was placed on a home built stand and a metal holder for the working electrode was designed and attached to the positioner. The current was measured by 
in-house built potentiostat that was interfaced with a computer through an ADC card (Talisman electronics, PCI-DAS6040) and software written in-house. The current (or hydrophone response) was also recorded by a Tektronix TDS 2014 4-channel 100 MHz oscilloscope that was triggered by a photodiode (Thorlabs) placed close to the beam path. A schematic of the experimental arrangement is shown in figure 1 frame (u).

The solutions were made up using water from an USF Elga Purelab Option E10 water purification system. Water purified in this manner had a resistivity of at least $15 \mathrm{M} \Omega \mathrm{cm}$ and a low organic content $(\mathrm{TOC}<30 \mathrm{ppb})^{1}$. Sodium sulphate $(\mathrm{BDH}$, AnalaR), potassium ferrocyanide (Aldrich, 99\%) and potassium iodide (Timstar laboratory suppliers) were used as received. The electrolyte was filtered through a 0.2 $\mu \mathrm{m}$ to remove particulate matter and degassed prior to the electrochemical experiments.

A Photosonics Phantom v7 high-speed video camera (borrowed from EPSRC instrument pool) was also employed in the experiments. The camera recorded images with the maximum speed $150000 \mathrm{fps}$ and used SR-CMOS sensors with a maximum resolution of $800 \times 600$ pixels at up to 4,800 fps giving 1.2 seconds of recording time. The resolution decreases as the recording speed increases, giving the resolution of 32 x 32 pixels at the highest recording speed $(\sim 150,000 \mathrm{fps})$. A Pentium 4 PC that was connected to camera processor via 10/100 Ethernet controlled the camera. The camera recorded continuously and therefore both pre and post-trigger images were available. Macro lenses were used to focus the camera to the target. The images could be saved

\footnotetext{
${ }^{1}$ manufacturer quoted figure
} 
in a TIF-form and they could be downloaded and edited with the Cineview-software provided. The high-speed camera was also triggered by the photodiode.

A G.R.A.S. 10DB needle hydrophone that measures high frequencies (1-20 $\mathrm{MHz}$ with $< \pm 2 \mathrm{~dB}$ over this range $\mathrm{e}^{2}$ ) and high pressure amplitudes (up to $100 \mathrm{MPa}^{3}$ ) was employed to detect the cavitation events. It is within these limits that the hydrophone calibration is valid. A more significant restriction is the sampling frequency of $5 \mathrm{MHz}$. The hydrophone signals are impulsive and, as will be shown in Figures 3(b) and 4(b), the largest signals detected at the position of the hydrophone were around $3 \mathrm{MPa}$, and corresponded to the shock emitted as the liquid is broken down by the focussed laser action (denoted $\mathrm{LS}_{2}$ in Figure 4 (b)). As expected for such impulsive signals, their spectral content is broadband (see figure 4 (b) insert), and as the hydrophone signal was used only to note the timing of the arrival of the impulsive pressure wave at the hydrophone, then this will be achieved with high accuracy by measurement at one position by this hydrophone.

In order to avoid damage, the hydrophone was placed at least $3 \mathrm{~mm}$ away from the focal spot of the laser beam where the bubbles were generated.

\footnotetext{
${ }^{2}$ obtained from manufacturer

${ }^{3}$ manufacturer quoted figure
} 


\section{Results and Discussion}

Figure 1 shows the shape and size of a bubble event created in the apparatus used to study single cavitation bubbles generated by laser pulse. In this set of frames it can be seen that the bubble is generated below the electrode substrate (highlighted in figure 1 frame (a)). The bubble can be seen to grow over a period of $\sim 60 \mu$ s and then collapse between frame (h) and (i) (after $\sim 110 \mu \mathrm{s}$ ). Bubble rebound is observed through frames (i) to (n), after which time the bubble fragments leaving a cloud of small bubbles close to the electrode surface. In addition to this growth and collapse phases of the bubble, it is observed that the bubble moves towards the microelectrode. This motion can be attributed to both buoyancy and Bjerkness forces [1] driving the bubble towards the microelectrode. Jetting onto the surface appears most prominent in frame (l) after the primary collapse and rebound.

In order to discriminate between processes that are associated with the laser pulse (and its possible effects on the electrode surface) a number of experiments were performed to investigate the effect of laser power on the electrochemical current time transients recorded. During these experiments it was noted that a strong audible acoustic signature (a distinct 'cracking' sound) could be observed only above a certain critical laser energy (controlled through adjustment of the Q switch delay used in the experiment). Considering the nature of the experimental arrangement employed, it is likely that this observation is associated with a threshold for the formation of a large cavity within the liquid and its subsequent collapse. This hypothesis was supported by observation of high-speed video images recorded above and below this critical threshold. Below the threshold for formation of the audible acoustic signature from the cell (which was also found to be dependant on the solution constituents), bubble 
formation was found not to occur as determined by either electrochemical or highspeed imaging data. For example figure 2 (a) shows the effect of laser pulse focussed into the liquid directly below a $25 \mu \mathrm{m}$ diameter Au microelectrode held in a solution containing a redox probe (specifically potassium ferrocyanide). In this case the electrode was held under mass transfer limiting conditions (+0.4 V vs. SCE). Hence, any perturbation in the diffusion field, caused by forced convection processes, will be detected as a positive current at the microelectrode. In the absence of such mechanisms, a steady state oxidation current of $\sim 10-14 \mathrm{nA}$ corresponding to the conversion of ferrocyanide to ferricyanide species at the electrode surface. This was observed at times $<-120 \mu$ s with respect to the laser pulse. It should be noted that the data acquisition was triggered by a photodiode which recorded the discharge of the laser at time $t=0 \mathrm{~s}$. In this experimental setup (gain, electrode and laser) the operation of the Q switch and the laser pulse causes a number of transients which operate over time $=-120 \mu \mathrm{s}$ to $\sim 220 \mu \mathrm{s}$. However, after this period the electrochemical response returns to a value close to the steady state current in the absence of the laser event. The exact origin of these transients is unknown but can be linked to the laser pulse. For example the positive transient prior to time $t=0 \mathrm{~s}$ corresponds to the laser $\mathrm{Q}$ switch delay. The events after $t=0 \mathrm{~s}$ also appear to be a result of the laser pulse ${ }^{4}$ as figure 2 (b) shows that there is no strong acoustic signature

\footnotetext{
${ }^{4}$ Note that these 'interference transients' appear prior to and after the laser pulse (indeed appear at the start of the Q switch delay), are dependant on the experimental apparatus employed, the gain of the electrochemical equipment and without solution breakdown to form a bubble or associated shockwaves. Hence, we can conclude that some of these interference effects are due to pickup from the laser. Nevertheless further investigation of other real effects (for example pressure waves and
} 
for solution breakdown and in turn no associated bubble emission. However, after $\sim 200 \mu$ s the current returns to a value close to, but in excess of, the steady state current. In the absence of shocks and bubble events, one could postulate that this slight increase in current could be the result of local solution heating as a consequence of laser pulse into the liquid. However, an accurate estimation of this effect is difficult to give as the exact energy lost as heat in the locality of the laser focal point is not known under the conditions employed. Nevertheless, the electrochemical data suggests that the perturbation of the mass transfer environment around the microelectrode is minimal under these conditions.

Figure 3 shows the effect of increasing the laser energy (by changing the Q switch delay) on both the electrochemistry and the acoustic emission within the liquid. In this case a strong audible acoustic emission was observed. In turn the data recorded from the hydrophone suggests that liquid breakdown as the result of laser action occurs (see figure 3 (b) $\mathrm{LS}_{1}$ ). In addition to the shock generated by laser pulse, a second and third acoustic event should be noted (labelled $S_{1}$ and $S_{2}$ respectively). This is attributed to a large cavitation bubble formation by the laser pulse at time $t=0$ $\mathrm{s}$ which subsequently grows and collapses after $t \sim 160 \mu \mathrm{s}$. After the primary collapse phase a rebound (and possible collapse) is observed as a weak second (labelled $\mathrm{S}_{2}$ ) acoustic event. In turn the electrochemical data shows an enhancement in the mass transfer to the electrode surface after $\sim 250 \mu \mathrm{s}$. The resulting transient peaks at $t \sim 400$ $\mu$ s after which decay back to the steady state was observed. This decay process corresponds to the relaxation of the hemispherical diffusion field of the electrode after surface heating) may also have an influence. However, this is beyond the scope of this paper and is under further investigation. 
the perturbation by the bubble growth and collapse has occurred. Note that this process will operate over the ms timescale (considering the electrode size and redox system employed) and so extends beyond the time window monitored. Interestingly the electrochemical data suggests a single event rather than an event with rebound (as indicated by the acoustic measurements and the high-speed imaging of similar events shown in figure 1). This may be due to the sensitivity limit of the electrochemical data in comparison with the acoustic signal. It should also be noted that the threshold between the breakdown of the liquid (and the subsequent formation of a large cavitation bubble), and non-breakdown of the liquid, was found to be dependent on the solution constituents (see figure $1 v s$. figure 3 and 4 for example).

Figure 4 shows the electrochemical and acoustic data recorded as the laser power (and hence bubble size) is increased. Figure 4 (b) shows that a shock is emitted as the liquid is broken down by the focussed laser action (denoted $\mathrm{LS}_{2}$ ). In this case the magnitude of the $\mathrm{LS}_{2}$ is considerably bigger $(>2.5 \mathrm{MPa}$ ) than that shown in figure 2 (b) $\left(\mathrm{LS}_{1} \sim 0.8 \mathrm{MPa}\right)$. In addition the signal from the bubble collapse is also considerable $\left(\mathrm{S}_{3} \sim 0.9 \mathrm{MPa}\right)$ and the rebound signal $\left(\mathrm{S}_{4} \sim 0.55 \mathrm{MPa}\right)$ is clearly defined. Interestingly the bubble lifetime (defined here as the time taken from laser pulse to primary collapse) has increased in comparison to figure 3 (b) to $t \sim 208 \mu \mathrm{s}$ in agreement with the assertion that the bubble size has increased. The rebound event was found to occur at $t \sim 314 \mu$ s with respect to the laser pulse. The electrochemical data also shows interesting features not seen before. Figure 4 (a) shows two mass transfer enhancement events (denoted as $B_{1}$ and $B_{2}$ ) after the events associated with the laser pulse. The separation in time between these two mass transfer events was found to be of the order of $120 \pm 9 \mu \mathrm{s}$. This is in close agreement with the separation 
between primary collapse and rebound of the bubble $(106 \mu \mathrm{s})$ determined from the acoustic trace (note some degree of difference between these separate measurements is expected even though the laser power and other experimental conditions were kept the same). This evidence suggests that the events labelled $B_{1}$ and $B_{2}$ are associated with primary bubble collapse and rebound events respectively.

The mass transfer coefficients recorded from these experiments are of the order of $0.16 \mathrm{~cm} \mathrm{~s}^{-1}$. Considering that the rate constant for heterogeneous electron transfer has been reported for this system to be $0.3-0.4 \mathrm{~cm} \mathrm{~s}^{-1}$ [30] and that the potential of the electrode is on the mass transfer limiting plateau of the redox system (as determined from voltammetry not shown), we can assume that electrode kinetic effects will not play a significant role in the interpretation of the data.

Finally, it should be noted that due to the high gain (used to ensure a good signal to noise ratio for this system) employed $\left(10^{7} \mathrm{~V} \mathrm{~A}^{-1}\right)$, the response time of the current follower is likely to be limiting ${ }^{5}$. Hence the absolute current magnitude at the event peaks will be restricted by op-amp response. Clearly under these conditions conclusions regarding the absolute magnitude of the mass transfer enhancement require further investigation. This is currently underway.

It is interesting to note that the mass transfer enhancements (where strong rebound was observed - see figure 4) are most prominent after the primary collapse and rebound: whereas the acoustic signature as a result of bubble action is strongest

\footnotetext{
${ }^{5}$ The response time of the current follower employed was measured by applying a potential step across a dummy resistor $\left(1 \times 10^{7} \Omega\right)$ at a gain of $1 \times 10^{7} \mathrm{~V} \mathrm{~A}^{-1}$. The maximum response time was found to be $10.2 \pm 0.5 \mathrm{~mA} \mathrm{~s}^{-1}$ while the maximum response obtained from the current time trace (defined as the gradient of the steepest section of the $i-t$ trace) at $\sim 185 \mu \mathrm{s}$, figure 4 (a)) was $10.6 \pm 0.6 \mathrm{~mA} \mathrm{~s}^{-1}$.
} 
from the primary collapse (see $S_{3}$ ). This observation may indicate that the motion of the bubble towards the interface (observed in the high-speed video footage) may result in the secondary collapse of the bubble providing more mass transfer enhancement (and hence higher currents) when compared to the primary collapse. However, it should be noted that the video sequence shown in figure 1 was not recorded at the same time as the electrochemical data shown in figures 2 through 4 . Hence further evidence for this link can only be obtained if simultaneous video and electrochemical data is obtained. This investigation is currently underway. 


\section{Conclusions}

The mass transfer enhancement from a single large cavitation bubble has been measured using a microelectrochemical technique. Significant enhancements in mass transfer (mass transfer coefficients of up to $0.16 \mathrm{~cm} \mathrm{~s}^{-1}$ ) have been demonstrated when single bubbles are generated by laser pulse close to the electrode surface. Evidence for bubble growth and rebound has been gained from electrochemical, acoustic and high-speed video footage. A threshold in electrolyte solutions for the formation of large cavitation bubbles has been noted from both electrochemical and acoustic data.

\section{Acknowledgements}

We thank the EPSRC and MoD (JGS system) for funding for HMH (GR/S01764/01), The Engineering Loan pool for the use of the high-speed camera used in this work and Dr Graham Ball for useful discussions. 


\section{Figure legends}

Figure 1. High-speed video sequence of a single laser generated bubble below a microelectrode. Frame rate $=68,000 \mathrm{fps}$. The laser power was $\sim 9 \mathrm{~mJ}$. The solution consisted of $1 \mathrm{mM} \mathrm{KI}$ in $0.2 \mathrm{M} \mathrm{Na}_{2} \mathrm{SO}_{4}$. The experiments were performed at room temperature $\left(\sim 20{ }^{\circ} \mathrm{C}\right)$. The scale bar (see frame (a)) represents $1 \mathrm{~mm}$. The bubble maximum radius was measured at $535 \mu \mathrm{m}$ with an $s / R_{\max }$ value of 1.76 . Note the microelectrode (denoted by ' $M$ ') boundary is highlighted by the dotted line in frame (a). Frame (a) is assigned as time $t=0 \mathrm{~s}$ for the discussion shown in the text the times of each frame are given in $\mu \mathrm{s}$ ). Frame (u) shows a schematic representation of the lens arrangement described in the experimental section. Note PD is the photodiode, WE the working electrode, CE the counter electrode and RE the reference electrode.

Figure 2. (a) Plots showing the simultaneous measurement of the response of the photodiode and the electrochemical signal recorded by a microelectrode $(25 \mu \mathrm{m}$ diameter $\mathrm{Au}$ ) following a laser pulse into the solution The electrode was held at +0.4 V vs. SCE. (b) shows the acoustic trace detected with a G.R.A.S. 10DB needle hydrophone in comparison to the laser pulse (shown as the photodiode output, $\mathrm{E}_{\mathrm{PD}}$ ). The laser power was $\sim 7 \mathrm{~mJ}$. The electrolyte consisted of a filtered and degassed ${ }^{6} 5$ $\mathrm{mM} \mathrm{K} \mathrm{K}_{4} \mathrm{Fe}(\mathrm{CN})_{6} / 0.1 \mathrm{M} \mathrm{Sr}\left(\mathrm{NO}_{3}\right)_{2}$ solution. The microelectrode was positioned $\sim 1.5$ $\mathrm{mm}$ from the focal point of the laser. The experiments were performed at room temperature $\left(\sim 20^{\circ} \mathrm{C}\right)$.

\footnotetext{
${ }^{6}$ although the electrolyte was degassed prior to use, some gas reintroduction may occur over the time period of the experiment
} 
Figure 3. (a) Plots showing the simultaneous measurement of the response of the photodiode and the electrochemical signal recorded by a microelectrode $(25 \mu \mathrm{m}$ diameter $\mathrm{Au}$ ) following a laser pulse into the solution. The electrode was held at +0.4 V vs. SCE. (b) shows the acoustic trace detected with a G.R.A.S. 10DB needle hydrophone in comparison to the laser pulse (shown as the photodiode output, $\mathrm{E}_{\mathrm{PD}}$ ). The laser power was $\sim 23 \mathrm{~mJ}$. All other experimental details are reported in figure 2 legend.

Figure 4. (a) Plots showing the simultaneous measurement of the response of the photodiode and the electrochemical signal recorded by a microelectrode $(25 \mu \mathrm{m}$ diameter $\mathrm{Au}$ ) following a laser pulse into the solution. The electrode was held at +0.4 V vs. SCE. (b) shows the acoustic trace detected with a G.R.A.S. 10DB needle hydrophone in comparison to the laser pulse (shown as the photodiode output, $\mathrm{E}_{\mathrm{PD}}$ ). The laser power was $\sim 44 \mathrm{~mJ}$. The insert shows an FFT analysis (Power Spectral Density/AU) of the signal denoted $\mathrm{LS}_{2}$. Note the pressure time trace was sampled at 5 MHz. All other experimental details are reported in figure 2 legend. 


\section{References}

[1] T. G. Leighton, The Acoustic Bubble, Academic Press, London, 1994.

[2] D. G. Offin, An investigation of fast surface re-formation in the presence of inertial (transient) cavitation, University of Southampton, Southampton, 2006.

[3] T. G. Leighton, M. Farhat, J. E. Field, and F. Avellan, Journal of Fluid Mechanics, 480 (2003) 43.

[4] I. Hansson, V. Kedrinskii, and K. A. Morch, Journal of Physics D: Applied Physics, 15 (1982) 1725.

[5] I. Hansson and K. A. Morch, Journal of Applied Physics, 51 (1980) 4651.

[6] B. Vyas and C. M. Preece, Journal of Applied Physics, 47 (1976) 5133.

[7] A. Vogel, K. Nahen, D. Theisen, and J. Noack, IEEE Journal of Selected Topics in Quantum Electronics 2(1996) 847.

[8] Y. Tomita and A. Shima, Acustica, 71 (1990) 161.

[9] W. Lauterborn and C. D. Ohl, Ultrasonics Sonochemistry, 4 (1997) 67.

[10] O. Lindau and W. Lauterborn, Journal Of Fluid Mechanics, 479 (2003) 327.

[11] A. Philip and W. Lauterborn, Journal of Fluid Mechanics, 361 (1998) 75.

[12] R. H. Mellen, Journal Of The Acoustical Society Of America, 28 (1956) 447.

[13] N. Moriguchi, Journal of the Chemical Society of Japan, 55 (1934) 749.

[14] C. E. Banks and R. G. Compton, Chemia Analityczna, 48 (2003) 159.

[15] S. Floate, J. L. Hardcastle, E. Cordemans, and R. G. Compton, Analyst, 127 (2002) 1094.

[16] J. Kruusma, C. E. Banks, E. Lust, H. Keis, L. Nei, and R. G. Compton, Electroanalysis, 16 (2004) 596.

[17] J. Kruusma, L. Nei, J. L. Hardcastle, R. G. Compton, E. Lust, and H. Keis, Electroanalysis, 16 (2004) 399.

[18] J. Kruusma, C. E. Banks, L. Nei, and R. G. Compton, Analytica Chimica Acta, 510 (2004) 85.

[19] P. R. Birkin and S. Silva-Martinez, Journal of Electroanalytical Chemistry, 416 (1996) 127.

[20] P. R. Birkin and S. Silva-Martinez, Journal of the Chemical Society Chemical Communications, (1995) 1807

[21] E. Maisonhaute, P. C. White, and R. G. Compton, Journal of Physical Chemistry B, 105 (2001) 12087.

[22] P. R. Birkin, R. O'Connor, C. Rapple, and S. Silva-Martinez, Journal of the Chemical Society Faraday Transactions, 94 (1998) 3365.

[23] P. R. Birkin, D. G. Offin, and T. G. Leighton, Electrochemistry Communications, 6 (2004) 1174.

[24] S. Silva-Martinez, Applications of Ultrasound in Electrochemistry, University of Southampton, Southampton, 1997.

[25] P. R. Birkin, Y. E. Watson, and T. G. Leighton, Journal of the Chemical Society Chemical Communications, (2001) 2650.

[26] P. R. Birkin, D. G. Offin, P. F. Joseph, and T. G. Leighton, Journal Of Physical Chemistry B, 109 (2005) 16997.

[27] P. R. Birkin, D. G. Offin, and T. G. Leighton, Physical Chemistry Chemical Physics, 7 (2005) 530.

[28] P. R. Birkin, D. G. Offin, and T. G. Leighton, Wear, 258 (2005) 623.

[29] G. Denuault, Chemistry in Industry, 18 (1996) 678. 
[30] C. Beriet and D. Pletcher, Journal of Electroanalytical Chemistry, 361 (1993) 93. 

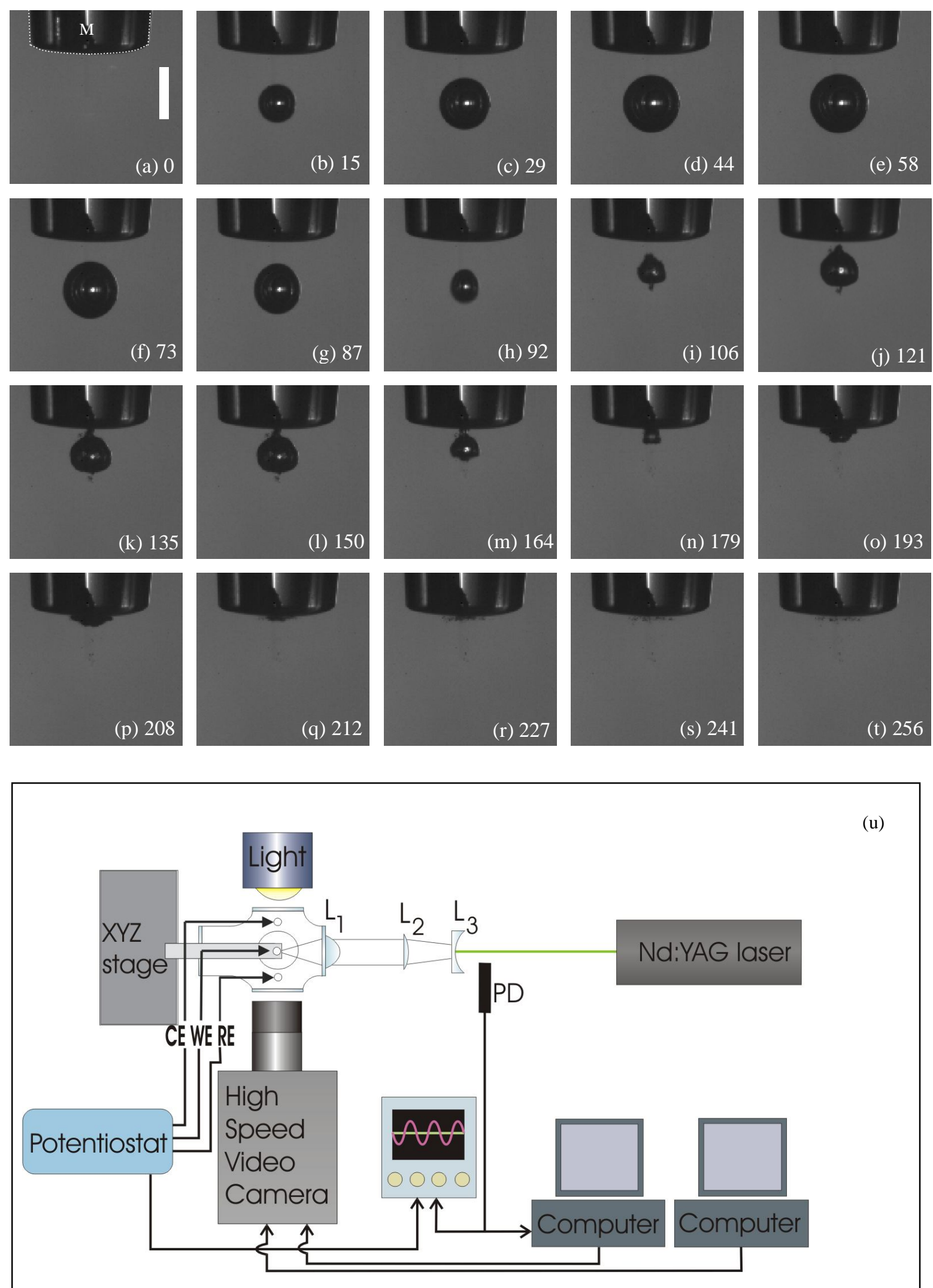

Figure 1 

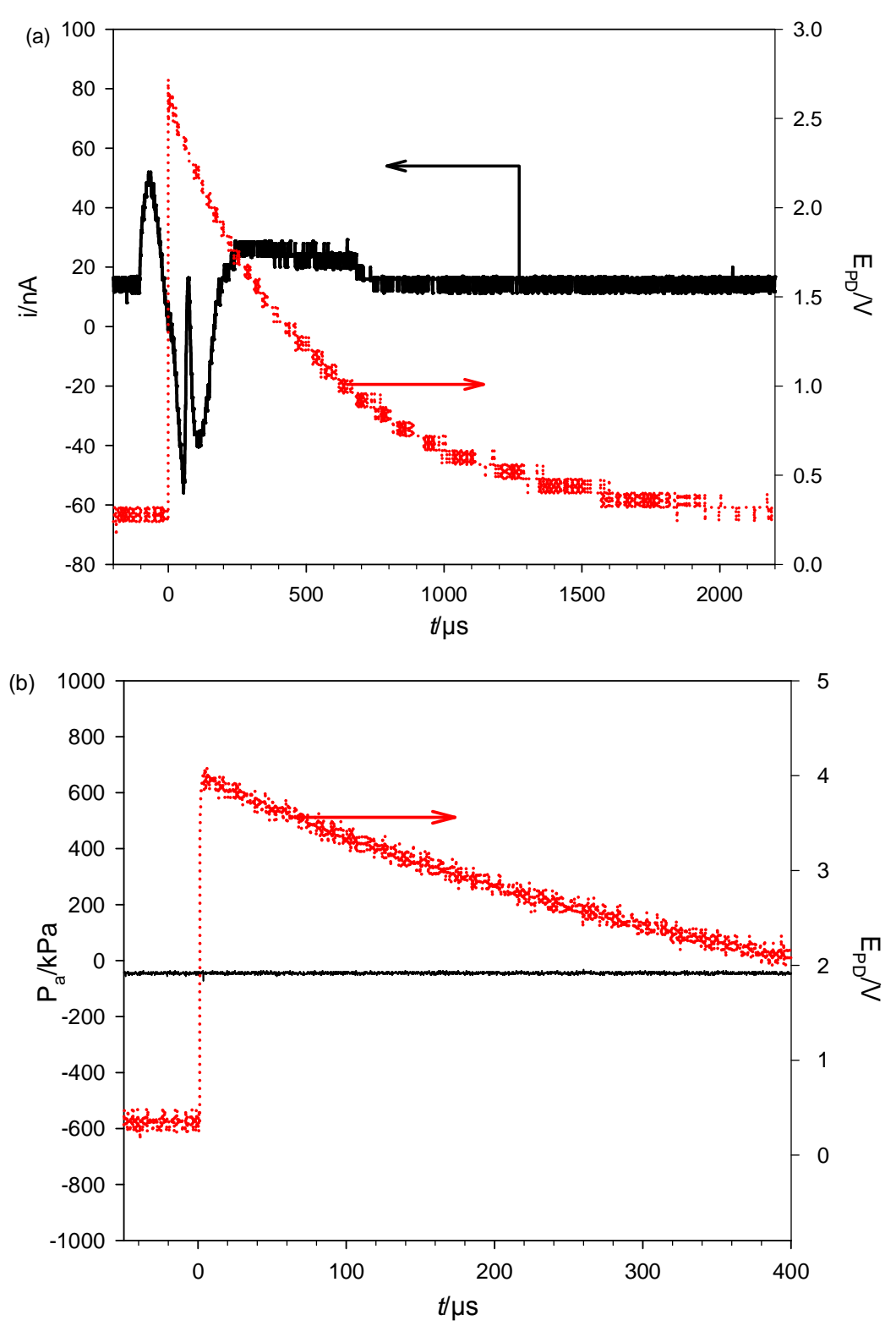

Figure 2 


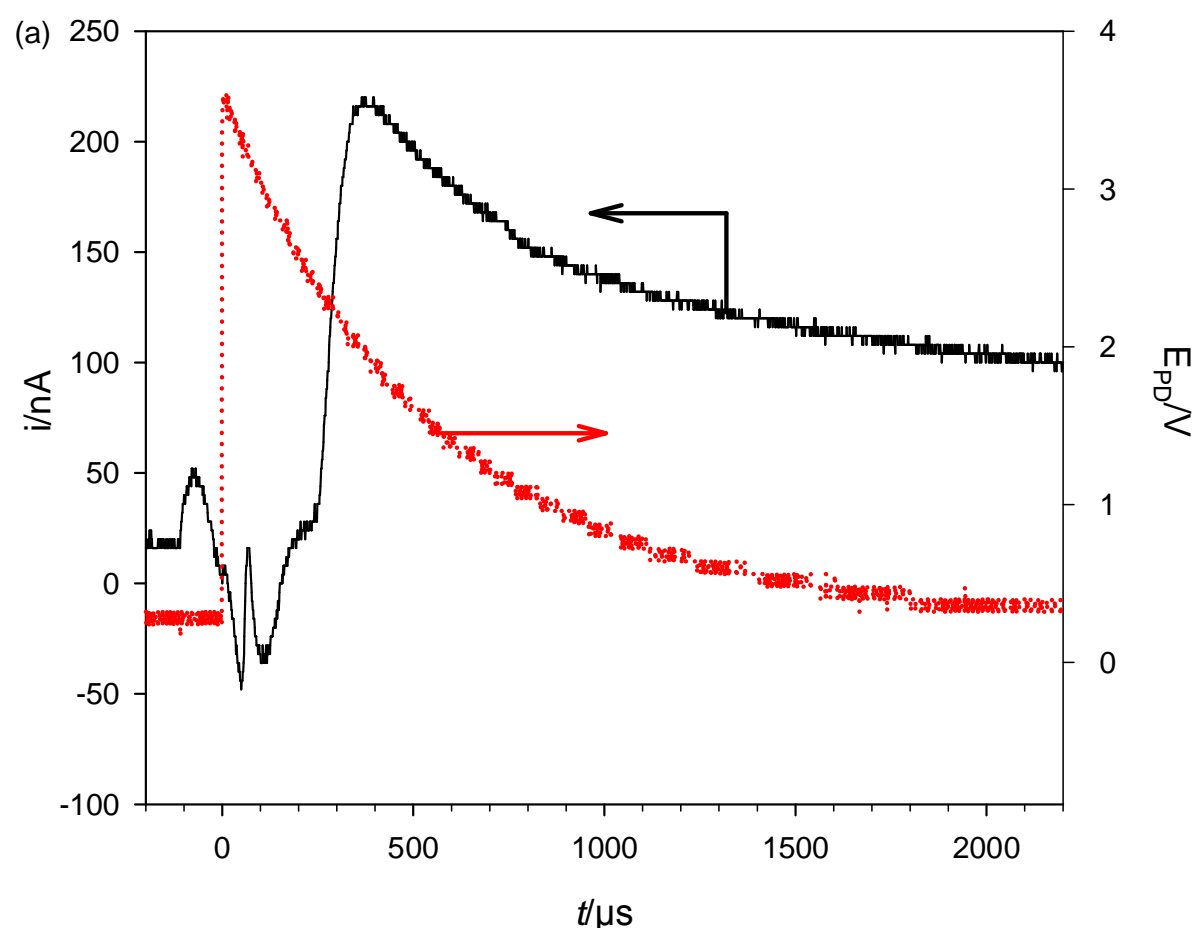

(b)

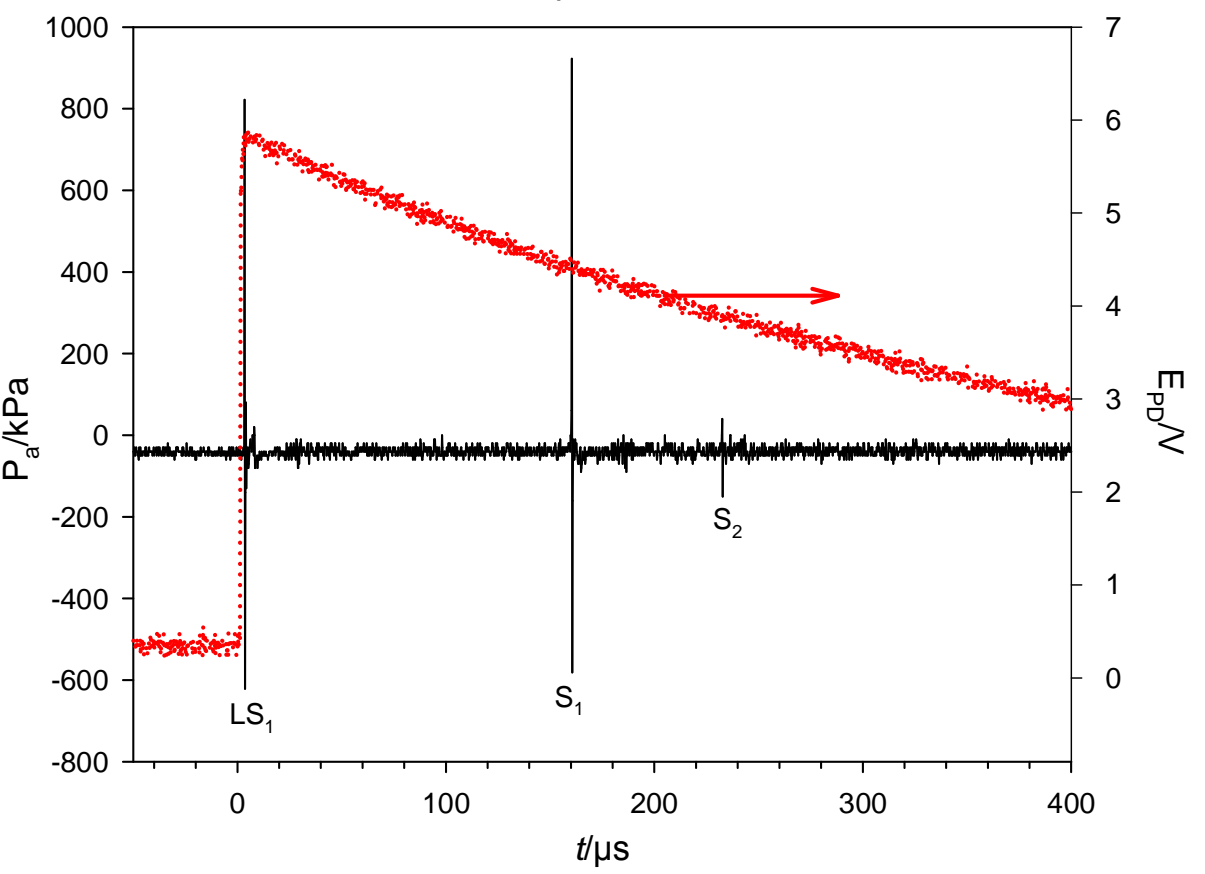

Figure 3 

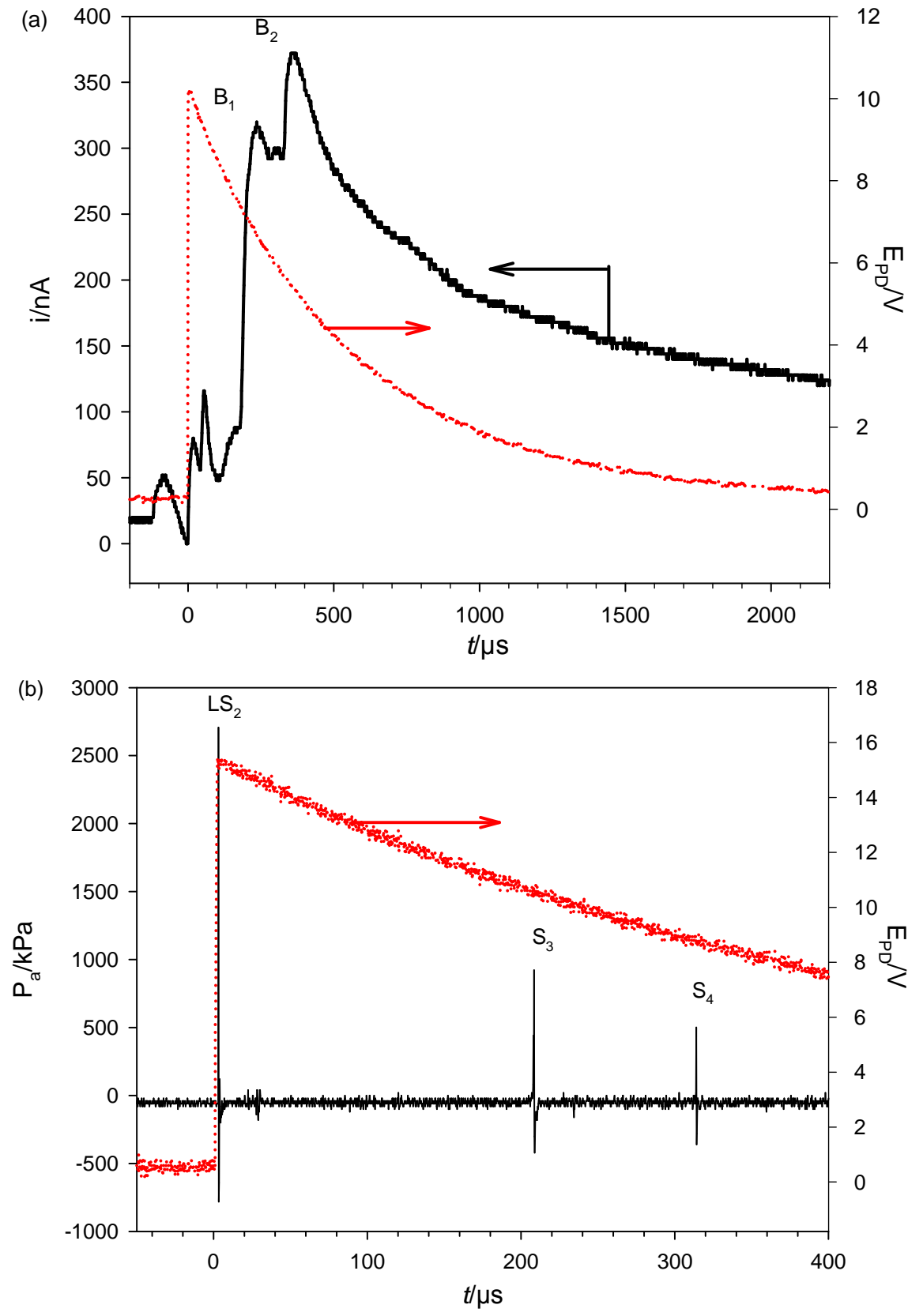

Figure 4 